\title{
Capturing non-Markovian dynamics on near-term quantum computers
}

\author{
Kade Head-Marsden $\odot,{ }^{1, *}$ Stefan Krastanov, ${ }^{1,2}$ David A. Mazziotti $\odot,{ }^{3}$ and Prineha Narang $\oplus^{1, \dagger}$ \\ ${ }^{1}$ John A. Paulson School of Engineering and Applied Sciences, Harvard University, Cambridge, Massachusetts 02138, USA \\ ${ }^{2}$ Department of Electrical Engineering and Computer Science, Massachusetts Institute of Technology, \\ Cambridge, Massachusetts 02139, USA \\ ${ }^{3}$ Department of Chemistry and The James Franck Institute, The University of Chicago, Chicago, Illinois 60637, USA
}

(Received 9 July 2020; revised 17 November 2020; accepted 10 February 2021; published 25 February 2021)

\begin{abstract}
With the rapid progress in quantum hardware, there has been an increased interest in new quantum algorithms to describe complex many-body systems searching for the still-elusive goal of "useful quantum advantage." Surprisingly, quantum algorithms for the treatment of open quantum systems (OQSs) have remained underexplored, in part due to the inherent challenges of mapping non-unitary evolution into the framework of unitary gates. Evolving an open system unitarily necessitates dilation into a new effective system to incorporate critical environmental degrees of freedom. In this context, we present and validate a new quantum algorithm to treat non-Markovian dynamics in OQSs built on the ensemble of Lindblad's trajectories approach, invoking the Sz.-Nagy dilation theorem. Here we demonstrate our algorithm on the Jaynes-Cummings model in the strong-coupling and detuned regimes, relevant in quantum optics and driven quantum system studies. This algorithm, a key step towards generalized modeling of non-Markovian dynamics on a noisy-quantum device, captures a broad class of dynamics and opens up a new direction in OQS problems.
\end{abstract}

DOI: 10.1103/PhysRevResearch.3.013182

\section{INTRODUCTION}

Open quantum systems (OQSs), quantum systems that are coupled to their environment, are ubiquitous in the physical sciences [1-10] and many classical techniques exist to describe the dynamics of an OQS beyond the Markov approximation [11-29]. While the Lindblad formalism gives an efficient and accurate depiction of the dynamics in the weak-coupling regime [30,31], the approach does not extend to systems that are strongly coupled to their environments [11,21]. Strong coupling can lead to non-Markovian effects such as recurrences of quantum properties, which are both important for a fundamental understanding of system dynamics and show promise for aiding in system control [32-40]. With the advent of quantum devices and the corresponding search for useful quantum advantage there has been an increased interest in algorithm development for physics and chemistry problems. Surprisingly, algorithm development for the treatment of open quantum systems has been limited to a few theoretical and experimental studies [3,41-46]. While there have been impressive recent strides in the field $[44,46]$, the lag in the development of this field is in part due to the challenge of the non-unitary evolution

\footnotetext{
*kheadmarsden@ seas.harvard.edu

†prineha@seas.harvard.edu

Published by the American Physical Society under the terms of the Creative Commons Attribution 4.0 International license. Further distribution of this work must maintain attribution to the author(s) and the published article's title, journal citation, and DOI.
}

of OQSs being cast into the framework of unitary quantum gates. To evolve an OQS unitarily, dilation methods must be used to incorporate the important environmental degrees of freedom into a new effective system. Early work faced computational scaling challenges with this dilation [42]; however, recent advances [47-49] have allowed accurate simulation of Lindbladian dynamics on a noisy-quantum device [44].

In this article, we present a quantum algorithm to treat non-Markovian dynamics in open quantum systems by extending the ensemble of Lindblad's trajectories method onto a quantum computer (ELT-QC) by invoking the Sz.-Nagy dilation theorem [44,47-49]. While previous work has looked at example systems for Markovian and non-Markovian dynamics on quantum computers, here we aim to provide the foundation for a universal and generalizable theory that allows for the investigation into open quantum system properties. We start by introducing the theory behind the ELT-QC method, followed by benchmarking the algorithm and demonstrating the impact of this approach on problems in quantum optics.

\section{THEORY}

\section{A. Ensemble of Lindbladian trajectories}

Density-matrix methods are a natural choice for modeling open quantum systems, and there have been a plethora of methods to capture Markovian and non-Markovian dynamics, from perturbative to numerical techniques [11-29,50-54]. However, in the non-Markovian regime, many of these methods struggle from the same challenges such as maintaining the positivity, and therefore physical nature, of the system density matrix. The ensemble of Lindblad's trajectories method 


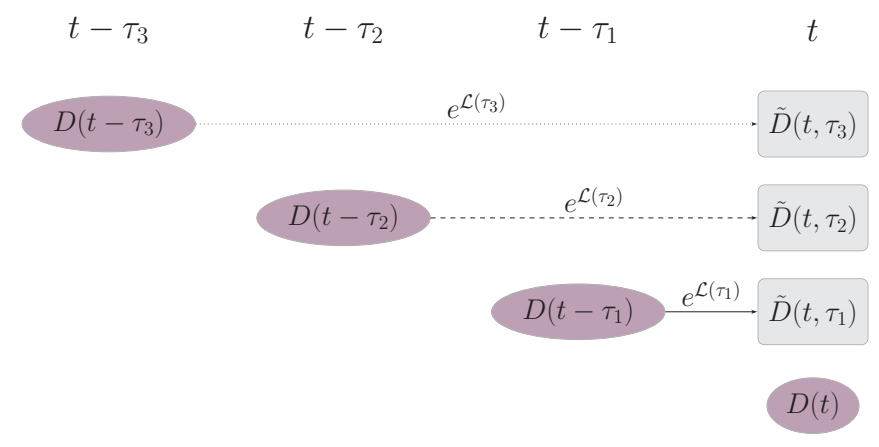

FIG. 1. An ensemble of Lindbladian trajectories whose weighted ensemble produces the true density matrix at time $t$.

is a recently developed, formally exact method depicted in Fig. 1, where true density matrices and auxiliary density matrices at time $t$ are represented as purple and gray, respectively, and the variable $\tau$ represents the time lag [23]. The ELT method extends the Lindbladian formalism beyond the Markovian regime through the use of an ensemble of trajectories originating from different points in the system's history [23]. Due to the relationship between Lindblad operators and Kraus maps [55], the density matrix remains positive semidefinite for all time, and, due to the ensemble average, non-Markovian behavior is captured. This method was also generalized to treat systems of multiple fermions with accurate statistics [24]. In the simplest discrete form this is mathematically equivalent to writing the density matrix as

$$
D(t)=\sum_{i=1}^{T} \omega\left(\tau_{i}\right) e^{\mathcal{L}\left(\tau_{i}\right)} D\left(t-\tau_{i}\right),
$$

where $T$ is the maximum memory, $\omega\left(\tau_{i}\right)$ are the statistical weights of the $i$ th trajectories, and $e^{\mathcal{L}\left(\tau_{i}\right)}$ are the propagators. Each trajectory is a Kraus map which we can represent by the following Lindbladian trajectory:

$$
\begin{aligned}
\frac{d D}{d s} & =\mathcal{L} \circ D \\
& =-i[H, D]+\sum_{i=1}^{\mathcal{M}} C_{i} D C_{i}^{\dagger}-\frac{1}{2}\left\{C_{i}^{\dagger} C_{i}, D\right\},
\end{aligned}
$$

where $s$ represents an effective time within the mapping, and the Lindbladian superoperator $\mathcal{L}$ can be written in terms of the system Hamiltonian $H$ and Lindbladian matrices $C_{i}$, which account for the interaction of the reduced density matrix with its environment through $\mathcal{M}$ different channels [30]. From the properties of Kraus maps the trajectories produce positivesemidefinite density matrices whose ensemble is also positive semidefinite [55].

In the continuous limit, this formulation can be related to a simplified form of the generalized master equation [23], where the traditional memory kernel is represented by the ensemble weights, the Lindbladian superoperator, and a time derivative. In this representation, just as in traditional use of Lindblad's equation, there are multiple avenues of approach to parameter selection, including but not limited to numerical optimization, extraction from short-time propagations, and use of experimental data.

\section{B. Quantum algorithm}

Recent work by $\mathrm{Hu}$ et al. has been done to map the Lindblad equation into a dilated unitary evolution, following which they performed this unitary evolution on a quantum computer [44]. In this work, the Lindblad equation is first written in operator sum form,

$$
D(t)=\sum_{i} M_{i} D M_{i}^{\dagger},
$$

where the $M_{i}$ are Kraus maps corresponding to the Lindbladian channels represented by each $C_{i}$ in the original equation. Since Kraus maps are contraction mappings of a Hilbert space, the Sz.-Nagy dilation theorem guarantees that there exists a related unitary operator dilation in a larger Hilbert space [44]. While different orders of $d$-dilation exist and correspond to contraction mappings being applied $d$ times to a Hilbert space, the 1-dilation is sufficient to consider either populations or coherence of a two-level system. Here, we focus on the 1-dilation which produces the unitary operator

$$
U_{M_{i}}=\left(\begin{array}{cc}
M_{i} & D_{M_{i}^{\dagger}} \\
D_{M_{i}} & -M_{i}^{\dagger}
\end{array}\right),
$$

where $I$ is the identity matrix and $D_{M_{i}}=\left(I-M_{i}^{\dagger} M_{i}\right)^{1 / 2}$.

To perform the unitary evolution, the density matrix is reformulated in vector form and dilated to account for the added environmental degrees of freedom. The density matrix can be represented as an ensemble of vectors $\left\{v_{j}\right\}$ where the vectors need not be orthogonal. The $\left\{v_{j}\right\}$ vectors are then dilated by padding with zeros to match the dimension of the unitary operator. In this work, only the population elements of the density matrix are considered. Given an ensemble of vectors $\left\{v_{j}\right\}$ and using this dilation, the population elements of the system are calculated as

$$
\rho_{k}=\frac{1}{2} \sum_{i, j}\left|\left(U_{M_{i}} \cdot v_{j}\right)[k]\right|^{2},
$$

where $\rho_{k}$ is the occupation number of the $k$ th state and the summation is over the dilations of all the Kraus operators $M_{i}$ acting on all the vectors $v_{j}$. Each term can be simulated in parallel, with circuits of gate count on the order of $n^{2}$, where $n$ is the dimension of the system [44].

Since the ELT method is an ensemble average of Lindbladian trajectories, the recent algorithm used to calculate Lindbladian trajectories on quantum devices can be used with the following generalization: In previous work when practically invoking the ELT method [23,24], each trajectory is written as

$$
\tilde{D}(t, \tau)=e^{\mathcal{L}} D(t-\tau),
$$

where the Lindbladian term $\mathcal{L}$ is a constant in terms of time, and $D(t-\tau)$ is the density matrix initialized from $\tau$ time steps in the system's history. This equation can be directly cast into the unitary dilation framework; however, it requires storage of each $D(t-\tau)$ along the way and the production of a new circuit describing each $D(t-\tau)$, or the production of an arbitrary state at each time point. While this is possible, it would be computationally taxing and preclude the possibility for a quantum speed up. 
Alternatively, using a variable change, we can shift the time dependence from the density matrix to the Lindbladian,

$$
\tilde{D}(t, \tau)=e^{\mathcal{L}(t-\tau, t)} D,
$$

where the Lindbladian term $\mathcal{L}$ depends on the time lag while $D$ is a constant density matrix. Instead of requiring an arbitrary state preparation at every step, this formulation requires only variation in the time input to produce the Lindblad matrices and therefore the unitary dilations. The ELT increases the computational cost of a single Lindbladian trajectory in Ref. [44] by a multiplicative prefactor of $T$, where $T$ is the number of trajectories in the ensemble average.

\section{RESULTS}

\section{A. Single Lindbladian trajectory}

The unitary evolution matrices of a two-level system in a single amplitude-damping Lindbladian channel are given by [44]

$$
U_{M_{0}}=\left(\begin{array}{cccc}
1 & 0 & 0 & 0 \\
0 & \sqrt{e^{-\gamma t}} & 0 & \sqrt{1-e^{-\gamma t}} \\
0 & 0 & -1 & 0 \\
0 & -\sqrt{1-e^{-\gamma t}} & 0 & -\sqrt{e^{-\gamma t}}
\end{array}\right)
$$

and

$$
U_{M_{1}}=\left(\begin{array}{cccc}
0 & \sqrt{1-e^{-\gamma t}} & \sqrt{e^{-\gamma t}} & 0 \\
0 & 0 & 0 & 1 \\
1 & 0 & 0 & 0 \\
0 & \sqrt{e^{-\gamma t}} & -\sqrt{1-e^{-\gamma t}} & 0
\end{array}\right),
$$

where $\gamma$ is the rate of decay. An initial density matrix of the form

$$
D(0)=\frac{1}{4}\left(\begin{array}{ll}
1 & 1 \\
1 & 3
\end{array}\right)
$$

is decomposed into an ensemble of dilated vectors [44]

$$
v_{0}=\left(\begin{array}{l}
0 \\
1 \\
0 \\
0
\end{array}\right)
$$

and

$$
v_{1}=\frac{1}{\sqrt{2}}\left(\begin{array}{l}
1 \\
1 \\
0 \\
0
\end{array}\right) .
$$

With this decomposition, the four necessary terms for each Lindbladian trajectory are calculated through the circuits shown in Fig. 2. The circuits were constructed and verified in both Qiskit [56] and QuTip [57,58]. It should be noted that the two qubit states follow the conventional notation where the vector $(1,0,0,0)$ represents both qubits in their ground states, $(0,1,0,0)$ represents $q_{0}$ in its excited state and $q_{1}$ in its ground state, $(0,0,1,0)$ represents $q_{0}$ in its ground state and $q_{1}$ in its excited state, and $(0,0,0,1)$ represents both qubits in their excited states.

Using these circuits, we consider a two-level system in an amplitude-damping channel with a system decay rate of (a)

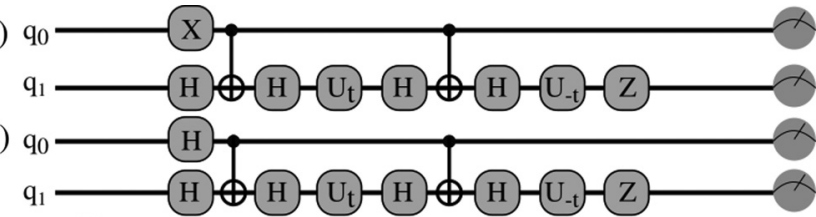

(c)
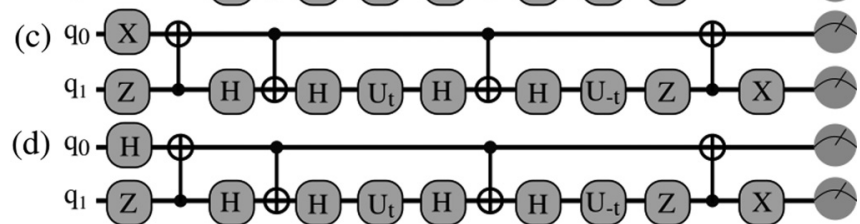

FIG. 2. Two qubit circuits are presented for the preparation of the following states: (a) $U_{M_{0}} v_{0}$, (b) $U_{M_{0}} v_{1}$, (c) $U_{M_{1}} v_{0}$, and (d) $U_{M_{1}} v_{1}$ where $X$ is the $\sigma_{x}$ gate, $Z$ is the $\sigma_{z}$ gate, $H$ is the Hadamard gate, $U_{t}$ is a rotation gate equivalent to $R_{y}(\theta)$ where $\theta=\cos ^{-1}\left[\left(\mathrm{e}^{-\gamma \mathrm{t}}\right)^{1 / 2}\right]$, and two-qubit gates are CNOT gates. The preparations are with respect to an initial state in which both qubits are in their ground states.

$\gamma=1.52 \times 10^{9} \mathrm{~s}^{-1}$. Using the solution from the Lindblad equation on a classical device, IBM's Qiskit simulator [56], and IBM's London device [59], the ground and excited-state populations, in purple and teal, respectively, are shown in Fig. 3. The solid lines represent the solution to the Lindblad equation on a classical device, the dots represent the result from the simulator, and the crosses result from the five-qubit London device. Both the simulator and device data agree well with the classical solution, producing accurate dynamics for a two-level system under Markovian conditions.

\section{B. Ensemble of Lindbladian trajectories}

Having verified this algorithm for a single trajectory of the Lindblad equation, we extend these circuits to treat the damped Jaynes-Cummings model, which consists of a single excitation in a two-level system coupled to a reservoir of harmonic oscillators $[11,50,60,61]$. This system is both exactly solvable and known to demonstrate non-Markovian

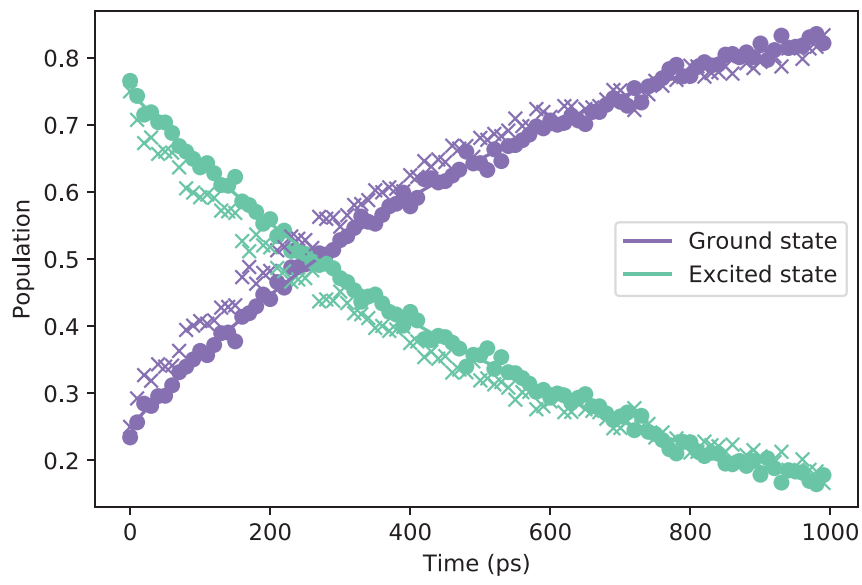

FIG. 3. Two-level system in an amplitude-damping channel where purple represents the ground-state population and teal the excited state population. The solid lines are the solutions on a classical device, the dots are generated using the IBM Qiskit simulator [56] and the crosses are generated using IBM's London device [59]. 


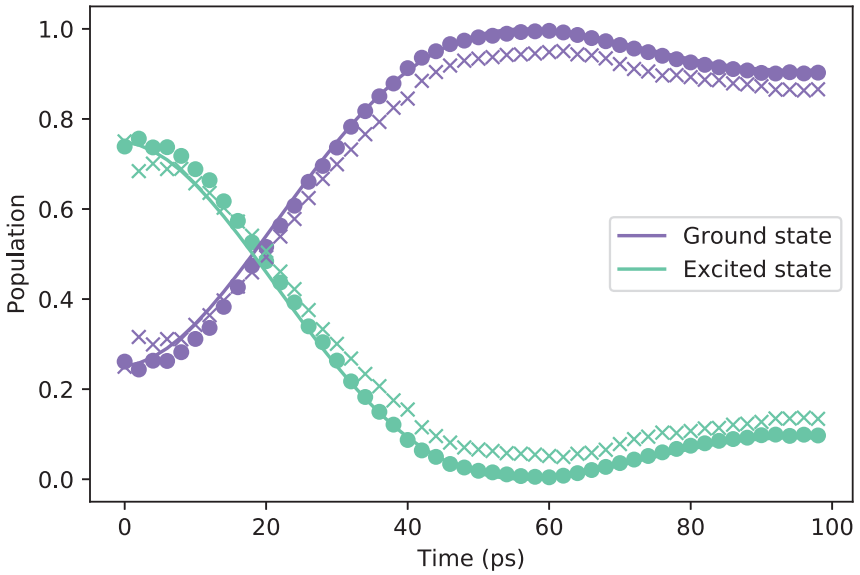

FIG. 4. Populations of the Jaynes-Cummings model in the strong-coupling regime, $\lambda=0.2 \gamma$, where purple represents the ground-state population and teal the excited state. The solid lines are the exact solution, the dots are generated using the IBM Qiskit simulator [56], and the crosses are generated using IBM's London device [59].

behavior in the strong-coupling and detuned regimes, making it an excellent benchmarking system for open quantum system methods $[11,23,60]$. The Hamiltonian is given by

$$
\hat{H}_{J C}=\hbar \omega \hat{a}^{\dagger} \hat{a}+\frac{1}{2} \hbar \omega_{0} \hat{\sigma}_{z}+\hbar \lambda\left(\hat{\sigma}_{+} \hat{a}+\hat{\sigma}_{-} \hat{a}^{\dagger}\right),
$$

where $\omega_{0}$ is the system's transition frequency, $\lambda$ is inversely proportional to the reservoir correlation time, $\hat{a}^{\dagger}$ and $\hat{a}$ are the creation and annihilation operators, respectively, and $\hat{\sigma}_{x}$, $\hat{\sigma}_{y}$, and $\hat{\sigma}_{z}$ are the Pauli spin operators with $\hat{\sigma}_{ \pm}=\hat{\sigma}_{x} \pm \hat{\sigma}_{y}$. In lieu of the cavity mode treatment, the bath spectral density is used [11],

$$
J(\omega)=\frac{1}{2 \pi} \frac{\gamma \lambda^{2}}{\left(\omega_{0}-\Delta-\omega\right)^{2}+\lambda^{2}},
$$

where $\omega_{0}$ is the system transition frequency, $\omega$ is the bath frequency, $\Delta$ is the detuning, and $\lambda$ is related to the system-bath coupling strength.

First we consider the strong-coupling case, where the bath relaxation parameter is given by $\lambda=0.2 \gamma$. In this regime, a single Lindbladian trajectory fails to capture accurate dynamics and a more involved method is required [11,50]. The populations of the Jaynes-Cummings model in the strongcoupling regime are shown in Fig. 4 using the ELT-QC method with weights numerically optimized as compared with the exact solution using Maple [62], as was done in Ref. [23]. The solid lines represent the exact solution while the dots and crosses are results from quantum simulation using the circuits shown in Fig. 2 using IBM's simulator and London device, respectively [56,59].

The ELT on a quantum simulator and device agrees well with the exact solution, demonstrating the ability of the ELTQC algorithm to accurately capture dynamics in the strongcoupling regime.

Next we consider the strong-coupling and detuned case, where $\Delta=\omega-\omega_{0} \neq 0$. In this regime, the bath relaxation parameter is given by $\lambda=0.3 \gamma$ and the detuning by $\Delta=2.4 \gamma$. The time-evolution of the populations are shown

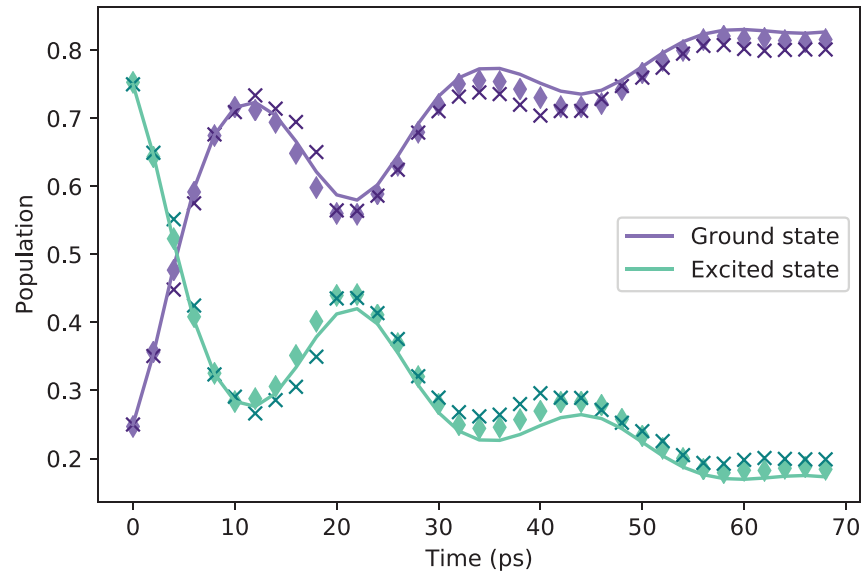

FIG. 5. Populations of the Jaynes-Cummings model in the strong-coupling and detuned regime, $\lambda=0.3 \gamma$ and $\Delta=2.4 \gamma$, where purple represents the ground-state population and teal the excited state. The solid lines are the exact solution, the dots are generated using the IBM Qiskit simulator [56], and the crosses are generated using IBM's London device [59].

in Fig. 5 where lines represent the exact result and the dots and crosses represent results from quantum simulation using the circuits shown in Fig. 2 using IBM's simulator and London device, respectively. These results are in excellent agreement with the exact solution, demonstrating that the ELT-QC algorithm captures accurate dynamics in the detuned regime for the Jaynes-Cummings model.

\section{CONCLUSIONS AND OUTLOOK}

Few algorithms exist for the treatment of open quantum systems on quantum devices, and existing algorithms are either restricted to Markovian systems or inconsistent with complete positivity. Our work presents an algorithm inspired by the ensemble of Lindblad's trajectories method [23] using an efficient dilation theorem [44] which allows for the treatment of non-Markovian dynamics of open quantum systems on a quantum device. The ELT-QC method is benchmarked on the Jaynes-Cummings model in the strong-coupling and detuned cases on both a quantum simulator and real fivequbit quantum computer, showing excellent agreement with the exact dynamics. While the Jaynes-Cummings model is a benchmark system, these results show our algorithm's ability to capture a wide variety of open quantum system dynamics accurately with tractable scaling. These regimes include strictly Markovian, or dissipative, dynamics and nonMarkovian dynamics induced by either strong or detuned system-environment coupling.

Moreover, this quantum algorithm retains the significant advantages of its classical counterpart, including an exact treatment of non-Markovian dynamics and complete positivity of the density matrices, meaning that the density matrices remain positive semidefinite with non-negative probabilities for all time. The ELT-QC algorithm offers a path towards polynomial time evolution of an electronic system in the presence of a complex environment. An accurate yet tractable description of open quantum systems on quantum devices has 
a myriad of significant applications from catalytic chemistry and correlated materials physics to descriptions of hybrid quantum systems and spin systems.

\section{ACKNOWLEDGMENTS}

The authors thank Dr. Evelyn M. Goldfield for the constructive feedback on the paper. This work is partially supported by the US Department of Energy, Office of Science, Basic Energy Sciences (BES), Materials Sciences and Engineering Division under FWP ERKCK47 "Understanding and Controlling Entangled and Correlated Quantum States in Confined Solid-state Systems Created via Atomic Scale Manipulation." K.H.M., S.K., and P.N. acknowledge sup- port from the Harvard Physical Sciences Accelerator Award and Harvard Quantum Initiative Seed Grant. D.A.M. gratefully acknowledges the US Department of Energy, Office of Science, Basic Energy Sciences (BES) under Grant No. DESC0019215, the National Science Foundation (NSF) under Grant No. CHE-1152425, and the U.S. Army Research Office Grant No. W911NF-16-1-015. P.N. is a Moore Inventor Fellow supported through Grant No. GBMF8048 from the Gordon and Betty Moore Foundation. We acknowledge the use of IBM Quantum services for this work. The views expressed are those of the authors and do not reflect the official policy or position of IBM or the IBM Quantum team. K.H.M., D.A.M., and P.N. also acknowledge the NSF RAISE-QACQSA, Grant No. DMR-2037783.
[1] C. Gardiner and P. Zoller, Quantum Noise: A Handbook of Markovian and Non-Markovian Quantum Stochastic Methods with Applications to Quantum Optics (Springer-Verlag, Berlin, Heidelberg, 2004).

[2] S. Maniscalco and F. Petruccione, Non-Markovian dynamics of a qubit, Phys. Rev. A 73, 012111 (2006).

[3] J. T. Barreiro, M. Müller, P. Schindler, D. Nigg, T. Monz, M. Chwalla, M. Hennrich, C. F. Roos, P. Zoller, and R. Blatt, An open-system quantum simulator with trapped ions, Nature (London) 470, 486 (2011)

[4] D. A. Mazziotti, Effect of strong electron correlation on the efficiency of photosynthetic light harvesting, J. Chem. Phys. 137, 074117 (2012)

[5] N. Skochdopole and D. A. Mazziotti, Functional subsystems and strong correlation in photosynthetic light harvesting, J. Phys. Chem. Lett. 2, 2989 (2011).

[6] R. Chakraborty and D. A. Mazziotti, Sufficient condition for the openness of a many-electron quantum system from the violation of a generalized Pauli exclusion principle, Phys. Rev. A 91, 010101(R) (2015).

[7] R. Chakraborty and D. A. Mazziotti, Noise-assisted energy transfer from the dilation of the set of one-electron reduced density matrices, J. Chem. Phys. 146, 184101 (2017).

[8] J. H. Fetherolf and T. C. Berkelbach, Linear and nonlinear spectroscopy from quantum master equations, J. Chem. Phys. 147, 244109 (2017).

[9] J. Flick, N. Rivera, and P. Narang, Strong light-matter coupling in quantum chemistry and quantum photonics, Nanophotonics 7, 1479 (2018).

[10] J. Flick and P. Narang, Cavity-Correlated Electron-Nuclear Dynamics from First Principles, Phys. Rev. Lett. 121, 113002 (2018).

[11] H. P. Breuer and F. Petruccione, The Theory of Open Quantum Systems (Oxford University Press, New York, 2002).

[12] A. Shabani and D. A. Lidar, Completely positive postMarkovian master equation via a measurement approach, Phys. Rev. A 71, 020101(R) (2005).

[13] A. Ishizaki and Y. Tanimura, Quantum dynamics of system strongly coupled to low-temperature colored noise bath: Reduced hierarchy equations approach, J. Phys. Soc. Jpn. 74, 3131 (2005).
[14] L. Chen, R. Zheng, Q. Shi, and Y. Yan, Optical line shapes of molecular aggregates: Hierarchical equations of motion method, J. Chem. Phys. 131, 094502 (2009).

[15] D. Chruściński and A. Kossakowski, Non-Markovian Quantum Dynamics: Local Versus Nonlocal, Phys. Rev. Lett. 104, 070406 (2010).

[16] A. Barchielli, C. Pellegrini, and F. Petruccione, Quantum trajectories: Memory and continuous observation, Phys. Rev. A 86, 063814 (2012).

[17] A. A. Budini, Post-Markovian quantum master equations from classical environment fluctuations, Phys. Rev. E 89, 012147 (2014).

[18] A. Montoya-Castillo, T. C. Berkelbach, and D. R. Reichman, Extending the applicability of Redfield theories into highly nonMarkovian regimes, J. Chem. Phys. 143, 194108 (2015).

[19] B. Vacchini, Generalized Master Equations Leading to Completely Positive Dynamics, Phys. Rev. Lett. 117, 230401 (2016).

[20] H.-P. Breuer, E.-M. Laine, J. Piilo, and B. Vacchini, Colloquium: Non-Markovian dynamics in open quantum systems, Rev. Mod. Phys. 88, 021002 (2016).

[21] I. de Vega and D. Alonso, Dynamics of non-Markovian open quantum systems, Rev. Mod. Phys. 89, 015001 (2017).

[22] S. Campbell, F. Ciccarello, G. M. Palma, and B. Vacchini, System-environment correlations and Markovian embedding of quantum non-Markovian dynamics, Phys. Rev. A 98, 012142 (2018).

[23] K. Head-Marsden and D. A. Mazziotti, Ensemble of Lindblad's trajectories for non-Markovian dynamics, Phys. Rev. A 99, 022109 (2019).

[24] K. Head-Marsden and D. A. Mazziotti, Satisfying fermionic statistics in the modeling of non-Markovian dynamics with oneelectron reduced density matrices, J. Chem. Phys. 151, 03411 (2019).

[25] V. Reimer, M. R. Wegewijs, K. Nestmann, and M. Pletyukhov, Five approaches to exact open-system dynamics: Complete positivity, divisibility, and time-dependent observables, J. Chem. Phys. 151, 044101 (2019).

[26] N. Yoshioka and R. Hamazaki, Constructing neural stationary states for open quantum many-body systems, Phys. Rev. B 99 , 214306 (2019). 
[27] A. Nagy and V. Savona, Variational Quantum Monte Carlo Method with a Neural-Network Ansatz for Open Quantum Systems, Phys. Rev. Lett. 122, 250501 (2019).

[28] M. J. Hartmann and G. Carleo, Neural-network Approach to Dissipative Quantum Many-Body Dynamics, Phys. Rev. Lett. 122, 250502 (2019)

[29] F. Vicentini, A. Biella, N. Regnault, and C. Ciuti, Variational Neural-Network Ansatz for Steady States in Open Quantum Systems, Phys. Rev. Lett. 122, 250503 (2019).

[30] G. Lindblad, On the generators of quantum dynamical semigroups, Commun. Math. Phys. 48, 119 (1976).

[31] V. Gorini, A. Kossakowski, and E. C. G. Sudarshan, Completely positive dynamical semigroups of $N$-level systems, J. Math. Phys. 17, 821 (1976).

[32] R. Wu, A. Pechen, C. Brif, and H. Rabitz, Controllability of open quantum systems with Kraus-map dynamics, J. Phys. A: Math. Theor. 40, 5681 (2007).

[33] P. Rebentrost, I. Serban, T. Schulte-Herbrüggen, and F. K. Wilhelm, Optimal Control of a Qubit Coupled to a NonMarkovian Environment, Phys. Rev. Lett. 102, 090401 (2009).

[34] L. A. Pachón and P. Brumer, Mechanisms in environmentally assisted one-photon phase control, J. Chem. Phys. 139, 164123 (2013).

[35] E.-M. Laine, H.-P. Breuer, and J. Piilo, Nonlocal memory effects allow perfect teleportation with mixed states, Sci. Rep. 4, 4620 (2014).

[36] D. M. Reich, N. Katz, and C. P. Koch, Exploiting nonMarkovianity for quantum control, Sci. Rep. 5, 12430 (2015).

[37] P. M. Poggi, F. C. Lombardo, and D. A. Wisniacki, Drivinginduced amplification of non-Markovianity in open quantum systems evolution, Europhys. Lett. 118, 20005 (2017).

[38] L. B. Ho, Y. Matsuzaki, M. Matsuzaki, and Y. Kondo, Realization of controllable open system with NMR, New J. Phys. 21, 093008 (2019).

[39] N. Mirkin, P. Poggi, and D. Wisniacki, Entangling protocols due to non-Markovian dynamics, Phys. Rev. A 99, 020301(R) (2019).

[40] N. Mirkin, P. Poggi, and D. Wisniacki, Information backflow as a resource for entanglement, Phys. Rev. A 99, 062327 (2019).

[41] H. Wang, S. Ashhab, and F. Nori, Quantum algorithm for simulating the dynamics of an open quantum system, Phys. Rev. A 83, 062317 (2011).

[42] R. Sweke, I. Sinayskiy, D. Bernard, and F. Petruccione, Universal simulation of Markovian open quantum systems, Phys. Rev. A 91, 062308 (2015).

[43] S.-J. Wei, D. Ruan, and G.-L. Long, Duality quantum algorithm efficiently simulates open quantum systems, Sci. Rep. 6, 30727 (2016).

[44] Z. Hu, R. Xia, and S. Kais, A quantum algorithm for evolving open quantum dynamics on quantum computing devices, Sci. Rep. 10, 3301 (2020).
[45] H.-Y. Liu, Y.-C. Wu, G.-C. Guo, and G.-P. Guo, Construct stationary states ansatz of open quantum systems with parameterized quantum circuits, arXiv:2001.02552.

[46] G. García-Pérez , M. A. C. Rossi, and S. Maniscalco, IBM Q experience as a versatile experimental testbed for simulating open quantum systems, npj Quantum Inf. 6, 1 (2020).

[47] H. Langer, B. Sz.Nagy, and C. Foias, Harmonic Analysis of Operators on Hilbert Space. VIII + 387S. Budapest/Amsterdam/ London 1970. Akadémiai Kiadó/NorthHolland Publishing Company, J. Appl. Math. Mech. 52, 501 (1972).

[48] V. Paulsen, Completely Bounded Maps and Operator Algebras (Cambridge University Press, Cambridge, 2002).

[49] E. Levy and O. M. Shalit, Dilation theory in finite dimensions: The possible, the impossible and the unknown, Rocky Mt. J. Math. 44, 203 (2014).

[50] H.-P. Breuer, B. Kappler, and F. Petruccione, Stochastic wavefunction method for non-Markovian quantum master equations, Phys. Rev. A 59, 1633 (1999).

[51] T. Yu, L. Diósi, N. Gisin, and W. T. Strunz, Post-Markov master equation for the dynamics of open quantum systems, Phys. Lett. A 265, 331 (2000).

[52] J. M. Moix and J. Cao, A hybrid stochastic hierarchy equations of motion approach to treat the low temperature dynamics of non-Markovian open quantum systems, J. Chem. Phys. 139, $134106(2013)$.

[53] A. C. Y. Li, F. Petruccione, and J. Koch, Perturbative approach to Markovian open quantum systems, Sci. Rep. 4, 4887 (2014).

[54] I. Semina and F. Petruccione, The simulation of the nonMarkovian behavior of a two-level system, Phys. A: Stat. Mech. Applic. 450, 395 (2016).

[55] K. Kraus, States, Effects, and Operations: Fundamental Notions of Quantum Theory (Springer-Verlag, Berlin, Heidelberg, 1983).

[56] H. Abraham et al., Qiskit: An open-source framework for quantum computing (2019), doi:10.5281/zenodo.2562110.

[57] J. R. Johansson, P. D. Nation, and F. Nori, Qutip: An open-source python framework for the dynamics of open quantum systems, Comput. Phys. Commun. 183, 1760 (2012).

[58] J. R. Johansson, P. D. Nation, and F. Nori, Qutip 2: A python framework for the dynamics of open quantum systems, Comput. Phys. Commun. 184, 1234 (2013).

[59] ibmq_london, ibm quantum team, retrieved from https:// quantum-computing.ibm.com (2020).

[60] E. T. Jaynes and F. W. Cummings, Comparison of quantum and semiclassical radiation theories with application to the beam maser, Proc. IEEE 51, 89 (1963).

[61] S. Stenholm, Beyond the Jaynes-Cummings model, J. Phys. B: At. Mol. Opt. Phys. 46, 224013 (2013).

[62] Maple 2019 (Maplesoft, Waterloo, 2021). 